\title{
New Liver Imaging Techniques
}

\author{
Mukta D. Agrawal · Sheela Agarwal · \\ Jorge M. Fuentes-Oreego $•$ Koichi Hayano • \\ Dushyant V. Sahani
}

Published online: 17 October 2013

(C) Springer Science+Business Media New York 2013

\begin{abstract}
Over the past decade, major technological advances have occurred in all the imaging modalities that have provided a completely new perspective to hepatic imaging. Dual-energy computed tomography (CT) acquires images at two different photon energies concurrently, and permits material decomposition based on the energydependent attenuation profile of specific material. It provides both morphologic and functional information in the same study, and potentially benefits hepatic tumor imaging. Hardware and software improvements in magnetic resonance imaging (MRI) technology have enabled faster MRI scanning and improved image quality. In addition to several newer MRI pulse sequences, the introduction of a new hepatocyte-specific contrast agent, gadoxetate disodium, offers additional benefits for improved detection and characterization of focal lesions, and allows functional hepatic and biliary imaging. In this article, we discuss the recent advances in CT and MRI technology and their applications for improving hepatic imaging.
\end{abstract}

\author{
M. D. Agrawal · S. Agarwal · J. M. Fuentes-Oreego . \\ K. Hayano $\cdot$ D. V. Sahani $(\bowtie)$ \\ Abdominal Imaging and Interventional Radiology Section, \\ Department of Radiology, Massachusetts General Hospital, \\ 55 Fruit Street, White Building, Room 270, Boston, MA 02114, \\ USA \\ e-mail: dsahani@partners.org \\ M. D. Agrawal \\ e-mail: magrawal@partners.org \\ S. Agarwal \\ e-mail: sagarwal5@partners.org \\ J. M. Fuentes-Oreego \\ e-mail: jmfuentes@partners.org \\ K. Hayano \\ e-mail: khayano@partners.org
}

Keywords Liver imaging - Dual-energy CT of liver . Magnetic resonance imaging of liver - Gadoxetate disodium enhanced MRI - Iterative reconstruction . Low dose CT

\section{Introduction}

The liver is affected by various pathologies that maybe characterized as diffuse or focal as well as benign or malignant, each requiring different management. Although detection and characterization are two primary objectives of liver imaging, the new expectations from imaging has increased due to a better understanding of disease processes, as well as the availability of more refined and individualized treatment options. Imaging modalities currently being used to detect and characterize liver lesions, include ultrasonography, computed tomography (CT), and magnetic resonance imaging (MRI). Dramatic technologic advances have benefited both CT and MRI, in particular MRI with the advent of new tissue-specific contrast agent.

\section{Multi-detector CT (MDCT)}

MDCT is often the first imaging modality performed for the screening of liver pathology, owing to its wider availability, faster scanning speed and reproducibility. CT offers the best spatial resolution and ability to study the entire liver in a single breath-hold. Recent technical advances in MDCT technology allow for thinner-slice scan acquisition with isotropic voxel resolution that allows for high quality three-dimensional (3D) image reformation [1-3]. An increase in the number of detector rows per scanner (up to 
320) [4-6] and faster gantry rotation (0.27 s) [7] has enabled greater $z$-axis coverage in a single breath-hold with the potential to improve image quality and reduce radiation dose. Several image noise reduction algorithms have been introduced that improve and optimize data processing to allow for radiation dose reduction while maintaining image quality $\left[8,9,10^{\bullet}, 11\right]$. With the advancement in MDCT technology, wider availability of commercial software and greater levels of experience, CT perfusion (CTP) is increasingly being used for the assessment of tumor response to therapies [12]. Dual-energy CT (DECT) is another major advancement in CT technology that acquires images at two different photon energies and allows for material decomposition. DECT concurrently provides both morphological and functional information that has the potential for numerous oncologic applications in the liver $[13,14]$. A variety of image series can be post-processed from the DECT data set that potentially improve detection and characterization of liver lesions, particularly in the setting of cirrhosis.

\section{Low kV Imaging}

The detection of hypervascular liver tumors at contrastenhanced CT (CECT) is directly related to tumor-to-liver contrast to noise (CNR) ratio. Traditionally, a $120-140 \mathrm{kV}$ tube potential is used for liver imaging. At low $\mathrm{kV}$ (80-100), iodinated contrast material (CM) demonstrates higher attenuation values owing to the proximity to the $k$-edge of iodine (33.2 kiloelectron-volts [keV]) [13]. Low $\mathrm{kV}$ improves conspicuity of hypervascular liver lesions by increasing contrast between enhancing lesions and background liver, and therefore improves lesion detection (Fig. 1) [15, 16]. This benefit is also extended in the portalvenous phase for more confident detection of hypovascular lesions such as metastases. Radiation dose reduction is an added benefit with low $\mathrm{kV}$ scanning, since radiation dose is directly related to the square of the $\mathrm{kV}$. Several investigators have tested and validated the application of a low $\mathrm{kV}$ technique for improving the diagnosis of liver tumors while simultaneously reducing patient's radiation exposure by
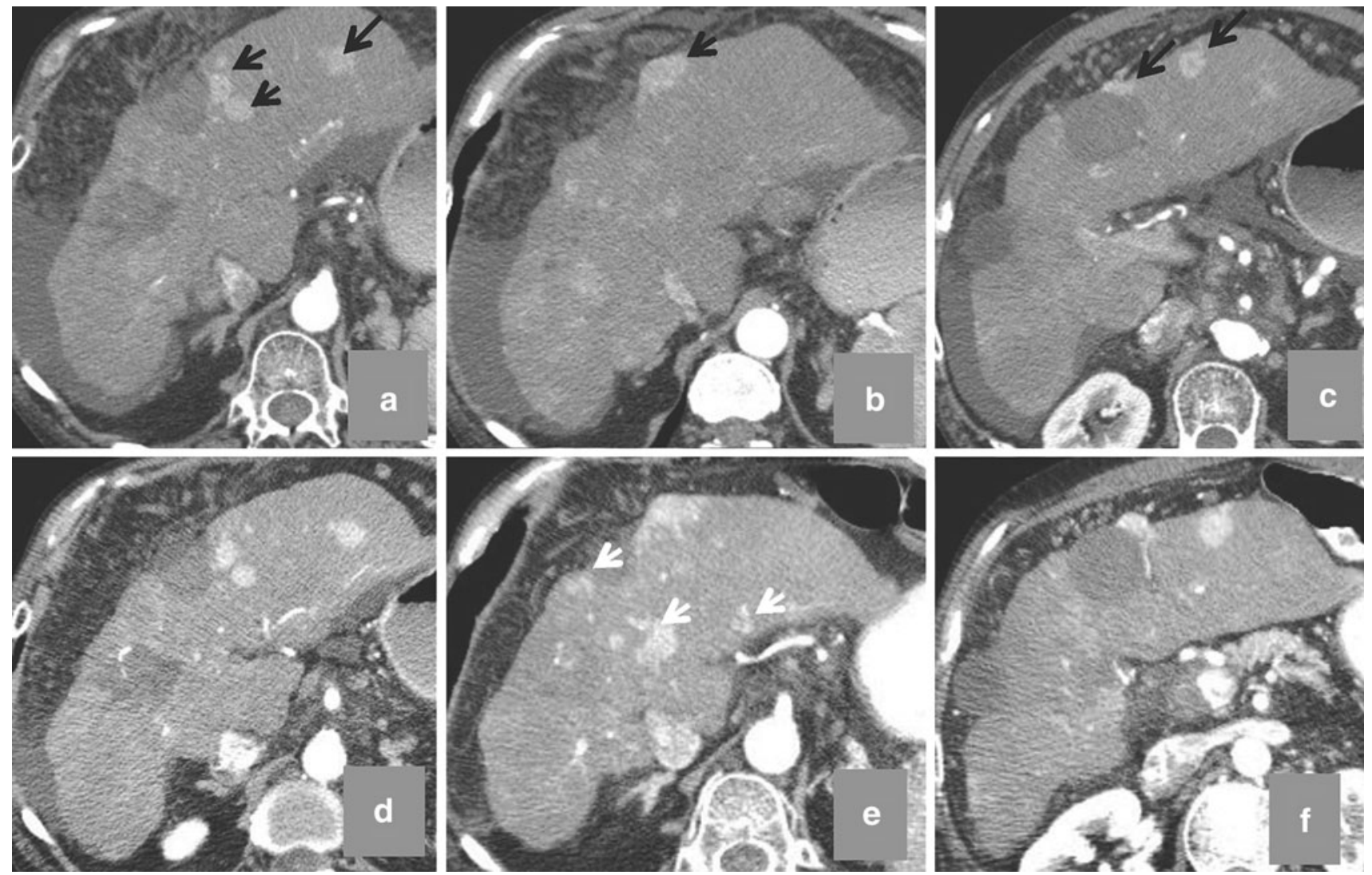

Fig. 1 Low kV imaging: a-f axial contrast-enhanced CT (CECT) from a 66-year-old-female (126 Ibs) with cirrhosis of liver. In comparison to initial screening study performed using $120 \mathrm{kV}(\mathbf{a}-\mathbf{c})$, the follow-up study performed 3 months later (d-f) using $100 \mathrm{kV}$ demonstrates higher conspicuity of enhancing liver nodules (black arrows). Additionally, few more enhancing nodules were identified (white arrows, e) on $100 \mathrm{kV}$ images. A $25 \%$ radiation dose reduction was achieved with $100 \mathrm{kV}$ technique $\left(\mathrm{CTDI}_{\mathrm{vol}}\right.$ of $8.36 \mathrm{mGy}$ using $100 \mathrm{kV}$ vs. $11.24 \mathrm{mGy}$ using $120 \mathrm{kV}$ ) 
40-50 \% [15-17]. However, use of low kV leads to corresponding increase in image noise, particularly in patients with a large body habitus ( $>200 \mathrm{Ibs}$ ) due to poor penetration, beam hardening artifacts and greater image noise, therefore requiring appropriate adjustments in other scan parameters. In general, we suggest using $80 / 100 \mathrm{kV}$ in smaller adult patients $(<150 \mathrm{Ibs} /$ body mass index [BMI] $20-25)$ and $100 / 120 \mathrm{kV}$ for average sized adults (151-100 Ibs/BMI 26-30), and to increase the tube current by $20-30 \%$. This can be achieved by either using a fixed high tube current or by using automated tube current modulation (ATCM) software. Scanners from different vendors work on different principles for ATCM, requiring knowledge of the technique as inappropriate adjustments may cause an unintended increase in radiation exposure. Due to space limitations, a detailed description of ATCM techniques is beyond the scope of this article and can be found in other review articles $[18,19]$.

\section{Iterative Reconstruction Technique (IR)}

Image noise reducing algorithms include conventional noise reduction filters, as well as novel approaches based on IR. These techniques alone do not reduce radiation exposure, but merely enhance the image quality of low dose CT by improving image noise and contrast. Thus, they indirectly enable CT dose reduction. Image noise reduction techniques can be concurrently used with low peak kilovoltage $(\mathrm{kVp}) \mathrm{CT}$ imaging to reduce noise and improve diagnostic image quality. The conventional filtered back projection (FBP) noise reduction technique has limitations in its ability to preserve diagnostic quality at low dose setting; therefore, major CT vendors have introduced IR technique to reduce noise and improve image contrast (Fig. 2). IR techniques include ASIR (adaptive statistical IR) from GE Healthcare, iDose from Philips Healthcare, SAFIRE (sinogram affirmed IR) from Siemens Healthcare, and adaptive iterative dose reduction from Toshiba. These reconstruction algorithms have been categorized as hybrid reconstruction techniques and work in image space. Image reconstruction for these algorithms occurs in real-time, and therefore does not pose a no major challenge for workflow. Several investigators have tested and validated the utility of such techniques that enable dose reduction by as much as $65 \%$ compared to standard abdomen and pelvis CT [8, 9, $11,20,21,22 \cdot, 23]$. At our institution, we routinely use an ASIR level of $30 \%$, an iDose level of 4 and a SAFIRE level of 3 for reconstruction of CT abdomen/pelvis, and achieve a $65 \%$ dose reduction over our standard FBP protocols. These new IR algorithms may thus have a significant impact, particularly on the imaging of young patients as well as patients requiring serial CT examinations (e.g., post-treatment follow-up exams in patients with hepatocellular carcinoma (HCC) and liver metastasis).

A recently introduced IR technique (Veo, GE Healthcare) works in the raw data domain to reduce image noise and preserve image quality. This model-based IR (MBIR) technique [10, 24], has shown tremendous potential with up
Fig. 2 Low-dose CT with iterative construction (IR): a-d axial and coronal CECT images from a middle-aged man (198 Ibs) with a hepatic hemangioma (white arrow) in right lobe of liver. a, b Latest study using $100 \mathrm{kV}$ and SAFIRE level 3 IR technique. Note increased conspicuity of the hemangioma on $\mathbf{a}$ and $\mathbf{b}$ in comparison with images $\mathbf{c}$ and d; the later were acquired using $120 \mathrm{kV}$. Images a and $\mathbf{b}$ have optimal image quality with $53 \%$ radiation dose reduction
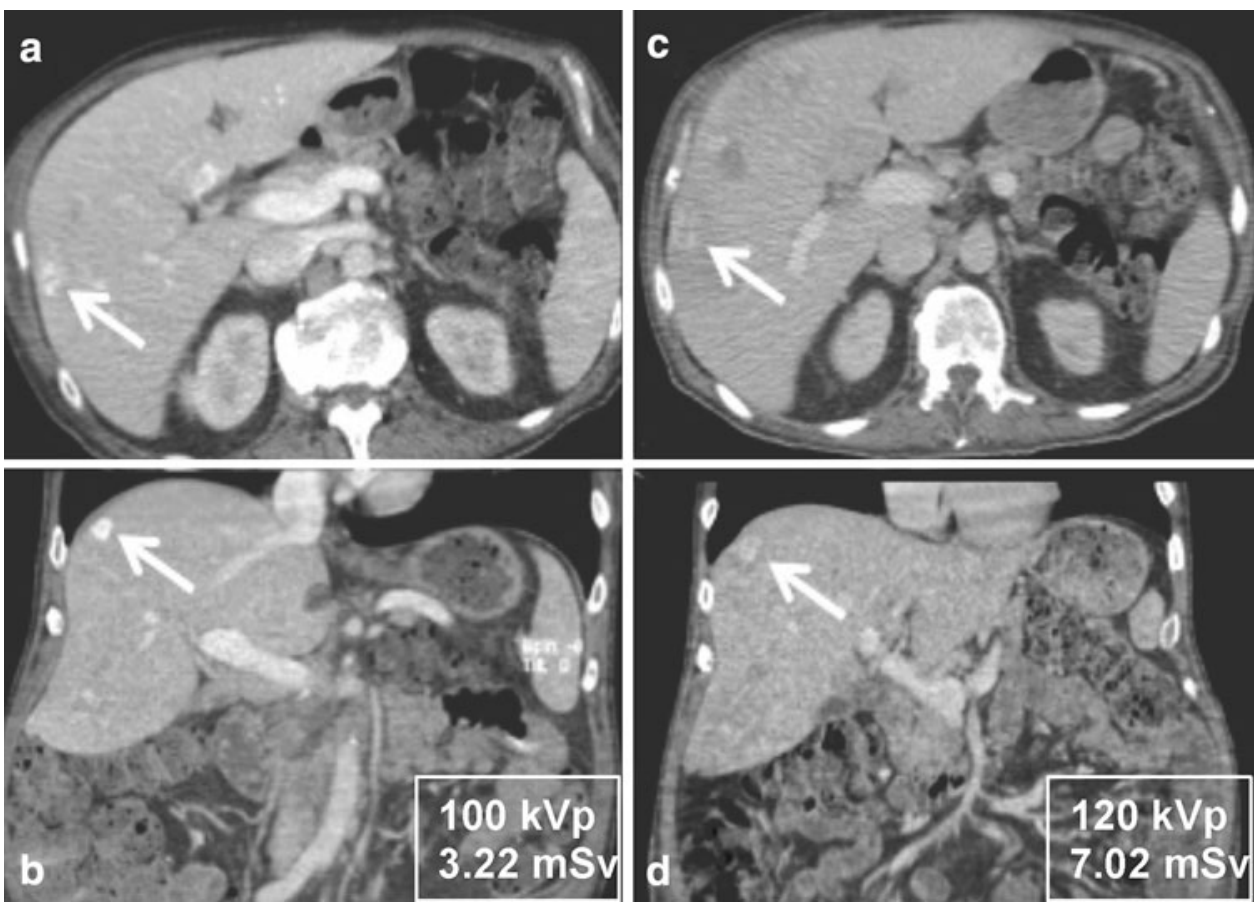
to $75 \%$ dose savings, but requires over 1 hour to reconstruct images of a single CT scan, thus severely limiting its implementation in clinical practice.

\section{Dual Energy CT}

DECT technology provides enhanced capabilities with potential application in hepatic tumor imaging. By virtue of scanning with two different energies, DECT permits material decomposition based on the energy dependent attenuation profile of specific materials [14]. The two DECT technologies currently available for clinical use are the dual source (ds) scanner and the single-source (ss) CT scanner with fast $\mathrm{kVp}$ switching [25]. Both technologies work on the same principle but use different approaches, and have different strengths and weaknesses (Table 1). The ds system uses two X-ray tubes and two arrays of detectors to simultaneously acquire images, while the ss system uses a single X-ray tube with fast $\mathrm{kVp}$ switching and energy separation performed at the detector level (Gemstone detector). Although their approaches and image post-processing algorithms are different, they both yield similar sets of post-processed images. DECT acquisition is relatively straightforward and can be performed by adapting existing institutional protocols used for conventional single-energy CT (SECT); however, extensive post-processing is performed on the DECT data set to generate images that are similar to SECT, as well as a variety of additional image series such as material density (MD) base pair images i.e. iodine density images and virtual un-enhanced (VUE) images, and virtual monochromatic (VMC) images.

\section{Iodine Density Images}

Iodine density images, as the name implies, display areas of tissue enhancement using iodinated CM. It detects and quantifies the amount of iodine within each voxel, and therefore even a small amount of enhancement within a lesion can be detected [25, 26•]. Iodine density maps also have the potential to increase detection of hypovascular metastases by increasing contrast between the hypoattenuating lesion and normally enhancing liver parenchyma, based on differences in tissue iodine content. Another potential application of iodine-specific maps is to improve detection of nodules with varying enhancement patterns in a cirrhotic liver. The iodine images are also useful in differentiating benign liver cysts from small metastatic liver lesions, as metastases with even a small amount of enhancement will reveal iodine, whereas the cysts remain dark (Fig. 3). While it eliminates the need for true unenhanced images (TUE) to assess tissue enhancement, imaging in different contrast media phases (arterial vs. portal-venous) is still necessary for specific hepatic lesion characterization.

Novel molecular targeted drug therapies such as antiangiogenesis drugs act by causing a change in tumor vascularity/viability and may not result in significant changes in tumor size. Quantification of tissue iodine on the iodine

Table 1 Characteristics of dual and single source DECT system

\begin{tabular}{lll}
\hline & Ds (dual source) & Ss (single source) \\
\hline Tube & Two & One (with fast kVp switching) \\
Multi-detector array & Two & One \\
Field of view for DE acquisition (cm) & $26-33$ & 50 \\
Automatic current modulation & Yes & No \\
Iterative reconstruction for noise reduction & IRIS or SAFIRE & ASIR (yes) \\
& & MBIR (no) \\
Post processing performed in & Image space (i.e. after the reconstruction & Projection space (i.e. before the reconstruction \\
& of high-energy and low-energy images) & of high-energy and low-energy images) \\
Post-processing method & Three material decomposition & Two material decomposition \\
& (Soft tissue, fat and iodine) & (Water and iodine) \\
Terminologies used for post-processed & & \\
$\quad$ images & & Virtual monochromatic (40-140) \\
Virtual monochromatic (VMC) images & Virtual monoenergetic (40-190) & Iodine images (mg/cm ${ }^{3}$ ) \\
$\begin{array}{l}\text { (range, keV) } \\
\text { (quaterial density iodine images }\end{array}$ & Iodine maps (Hounsfield units) & Water images \\
Simulated unenhanced images & & Virtual unenhanced (VUE) images
\end{tabular}

IRIS iterative reconstruction in image space, SAFIRE sinogram affirmed iterative reconstruction, ASIR adaptive statistical iterative reconstruction, MBIR model based iterative reconstruction 

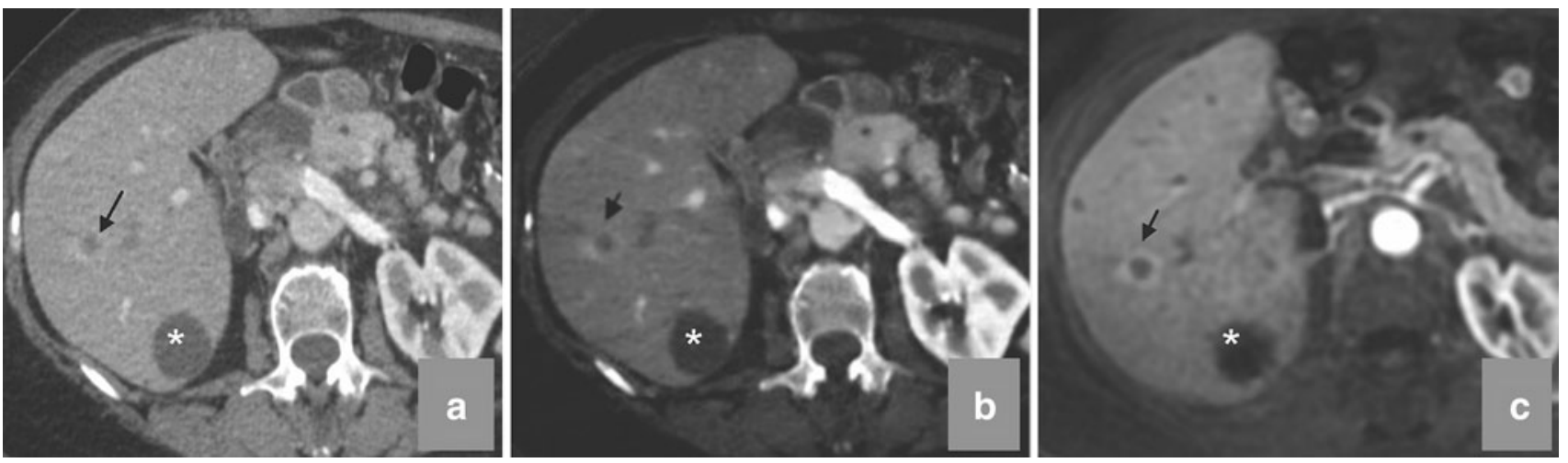

Fig. 3 Solitary liver metastasis and multiple cysts in a 67-year-old male with recent diagnosis of pancreatic adenocarcinoma (cyst vs. neoplasm): on CECT image (a), several hypodense hepatic lesions were identified in the liver and one of the lesions in the right lobe (arrow) was deemed suspicious due to faint rim enhancement. On the
MD iodine image (b), peripheral enhancement is more conspicuous (arrow) supporting the suspicion of metastasis. The rim-enhancing feature in this lesion was later confirmed on the contrast enhanced arterial phase T1-weighted fat-saturated MR image (arrow in c). Note simple cyst posterior to the metastasis $(*)$ density images can serve as an image biomarker of tumor viability beyond the conventional measurements of tumor size. In patients who undergo radio-frequency ablation of HCC or liver metastases, iodine images are useful to determine tumor viability, not only immediately after the procedure, but also on serial follow-up scans [27]. Although this approach still requires validation and standardization for use in clinical practice, it appears promising as a method for morphologic and functional evaluation of larger anatomic areas in one acquisition.

\section{Virtual un-enhanced (VUE) Images}

Since the behavior of iodine at different energies is known, iodine can be extracted from the image to generate a data set of simulated unenhanced images. These images can serve as a substitute for the TUE images, eliminating the need to acquire a separate unenhanced data set, and therefore reducing scan time and radiation burden. In the ss system, VUE are called water density images and quantification is done in terms of milligrams per milliliter. In the ds system, they are called virtual non-enhanced images and the quantification is done in Hounsfield units. Studies have confirmed that these simulated images can serve the intended purpose to reliably diagnose presence of small foci of calcium, fat and hemorrhage [28-30].

\section{Virtual monochromatic (VMC) Images}

Both types of DECT scanners can generate VMC from MD images using a complex mathematical algorithm. The VMC images depict objects as if they were imaged with a theoretical monochromatic beam, and the X-ray energy is reported as $\mathrm{keV}$ instead of $\mathrm{kVp}$. These single photon energy images provide more reliable CT attenuation values than conventional polychromatic CT images [31] by reducing beam-hardening artifacts [31]. VMC images of varying specific energies ranging from 40 to $140 \mathrm{keV}$ can be generated. The selection of the VMC energy should target the diagnostic task. In general, lower energy VMC images provide higher tissue contrast due to higher beam attenuation by iodine, but result in greater image noise, particularly in larger patients [13,31]. Conversely, higher energy VMC images provide less image contrast and less image noise. In general, 40-50 keV images provide highest $\mathrm{CNR}$ and are preferred for vascular imaging. However, 60-77 keV images provide appropriate balance of soft tissue contrast and noise and are generally considered most appropriate for the detection and characterization of hepatic lesions. The low $\mathrm{keV}$ images are good for detection of HCC and hypervascular liver metastases in the late arterial phase, and good for detection of hypovascular liver metastases in the portal-venous phase (Fig. 4) [32, 33]. Low $\mathrm{keV}$ images also have the potential to better delineate more precisely the tumor margin and its locoregional spread by increasing contrast between two adjacent tissues, thus providing better tumor staging [14].

\section{Liver Fat Quantification}

A recently introduced model-based method, known as 'multi-material decomposition' (air, fat, blood, calcium and iodine) algorithm, allows for quantification of liver fat content on enhanced DECT images. This algorithm has been tested in phantom and human studies and found to yield accurate results when compared to the current imaging standard (in-phase and out-of-phase T1-weighted liver MRI; [34]). This technique appears promising, but is 
Fig. 4 Hypervascular liver lesions: a, b axial CECT images in a patient with cirrhosis shows two hypervascular nodules in the liver (black arrow). On postprocessed axial virtual monochromatic images (VMC) reconstructed at $50 \mathrm{keV}(\mathbf{c}, \mathbf{d})$, the same lesions have improved conspicuity, and few additional lesions (white arrows in c) are better detected

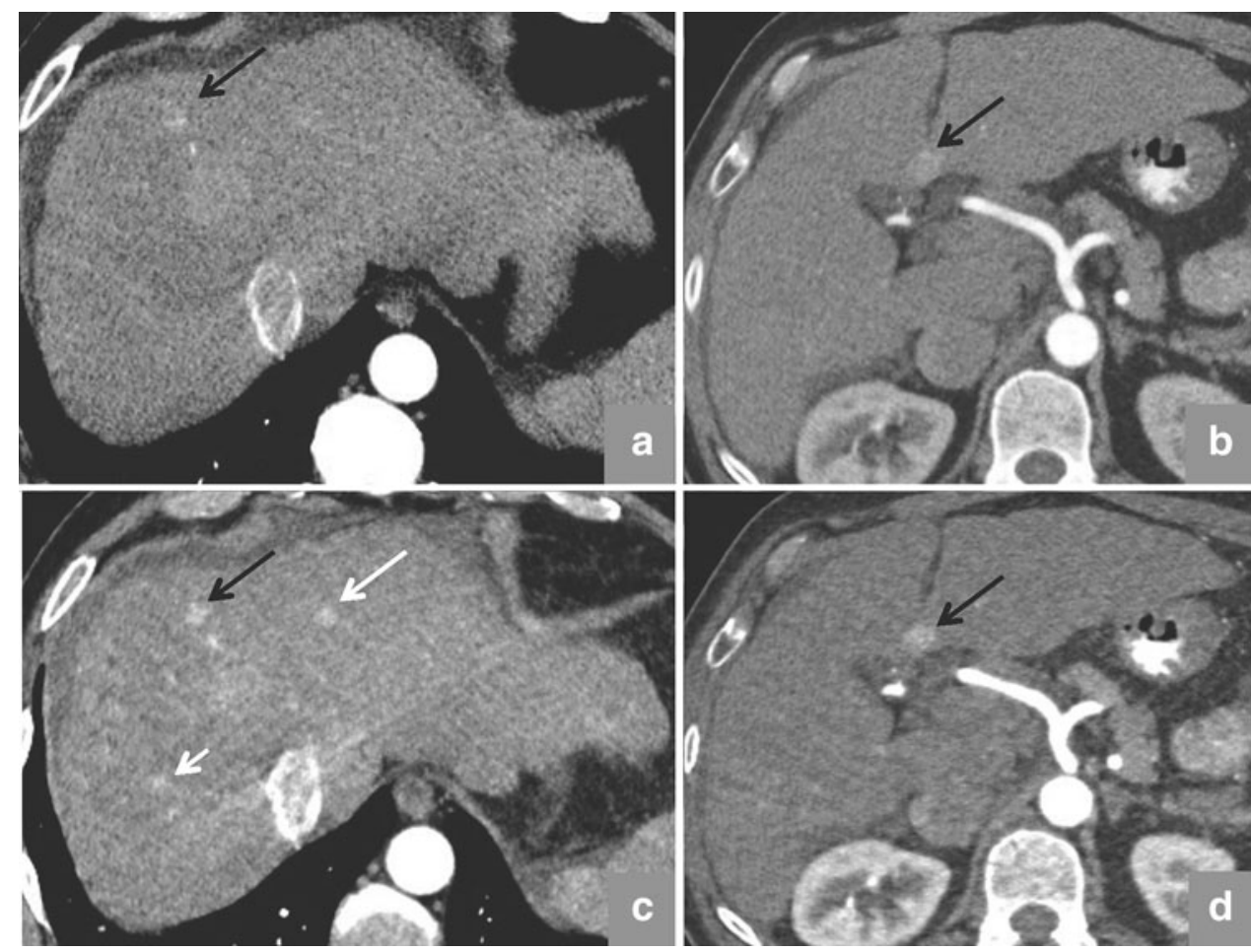

not yet Food and Drug Administration (FDA) approved for application in clinical practice and requires further validation and standardization.

\section{Post-operative Segmentation}

Partial liver resection and liver transplantation both require precise estimation of liver volume. This is crucial to ensure an adequate liver volume in the donor as well the recipient in the post-transplant setting, and to ensure adequate remnant liver volume in partial hepatectomy patients [35]. Technical advancements in fourth-generation MDCT scanners allow for thin-section data acquisition with isotropic voxel resolution that has improved the quality of post-processing algorithms such as maximum intensity projection and volume rendering for 3D display of vascular anatomy of liver donor, resulting in better accuracy and image quality for organ segmentation algorithms and subsequent volume estimation.

Tumor volumetric assessment has been recently proposed as a more accurate imaging biomarker for monitoring response to chemotherapy and radiotherapy and predicting long-term patient survival [36, 37]. Several researchers have highlighted the superiority of volume estimation for HCC and liver metastatic lesions over World Health Organization (WHO) and Response Evaluation Criteria In Solid Tumors (RECIST) criteria [38]. Manual segmentation on axial slices may be feasible for a single small-sized tumor; however, tumor volumetry of patients with more extensive tumor burden is time consuming and unsuitable for a busy radiology practice. Various computer assisted detection (CAD) and post-processing techniques have been developed for automated tumor recognition and subsequent tumor burden quantification. The MDCT data sets from fourth-generation scanners provide artifact-free CT data with superior voxel resolution. By providing clearer delineation of tumor margins and improved detection of tiny liver lesions, DECT ensures better performance of CAD applications [3]. Interpolation tools that calculate the volume between manually segmented slices and regiongrowing tools are two popular semi-automated methods for segmentation and volume estimation, since they allow for more operator control and increased accuracy [3].

\section{Perfusion CT}

CTP enables quantification of tumor vascularity by serially measuring temporal changes in tissue density following intravenous contrast administration. Since it was first described by Miles et al. [39], CTP has been successfully applied in a variety of clinical applications including assessment of liver cirrhosis [40, 41], characterization of liver tumors [42, 43], and evaluation of therapy response in liver malignancy [44].

Newer anticancer drugs such as anti-angiogenesis drugs (anti-VEGF), act by suppressing tumor growth by downregulating angiogenesis without causing any significant change in tumor size [44]. The traditional tumor response 
Fig. 5 CT perfusion (CTP) maps of a 65 -year-old-man with HCC treated with antiangiogenic drug: a, b axial CECT performed at baseline and at 2-weeks post-antiangiogenic treatment demonstrates no significant change in tumor enhancement (arrow) on CECT image. However, CTP analysis performed 2-weeks posttreatment (d) showed substantial reduction in tumor blood flow (BF; arrow) compared to baseline CTP (c), suggestive of response to treatment

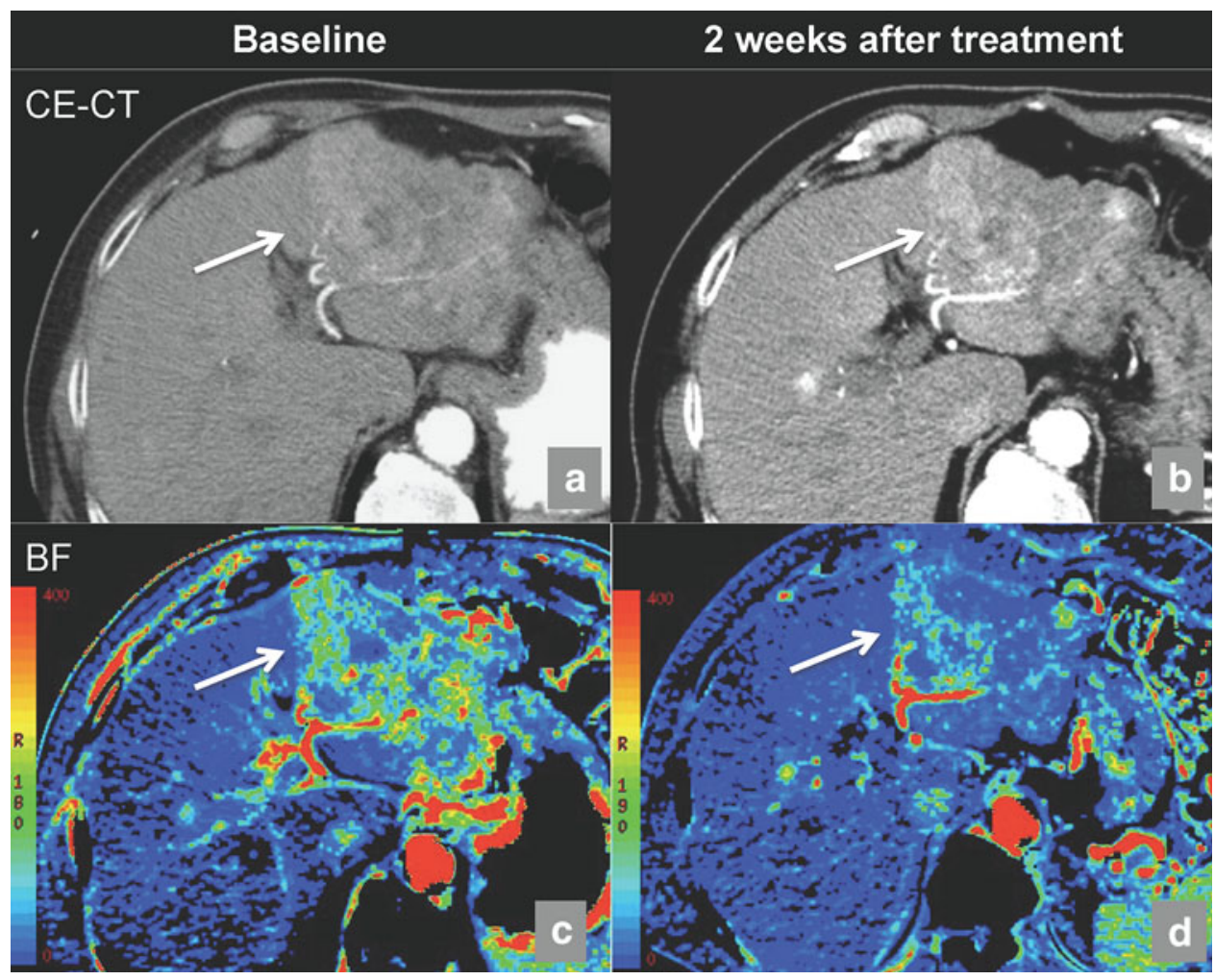

evaluation criteria (WHO and RECIST) do not take into account change in tumor vascularity/viability and can underestimate the effect of the newer molecular targeted therapies [45*•]. Thus, there is increasing interest to look for new imaging biomarkers that provide reliable and quantitative measurement of change in tumor vascularity. Conventional CT is a mainstay in oncologic evaluation, and is used for the diagnosis, staging and monitoring of anticancer therapies. CTP provides indirect measurement of tumor angiogenesis noninvasively, and can be readily incorporated into the existing CT protocols [46]. Most CT scanners now come equipped with sophisticated hardware platforms coupled with powerful and user-friendly software packages for CTP analysis. Perfusion parameters are dependent on the scan protocol and the mathematical model for perfusion analysis, but the commonly described CTP parameters include blood flow (BF), blood volume (BV), mean transit time (MTT), and permeability surface area product. Previous reports demonstrated that BF or BV are decreased as early as 2 weeks after initiation of antiangiogenic agents such as bevacizumab [44]. Therefore, perfusion imaging has the potential to be a biomarker of antiangiogenic therapy (Fig. 5). Jiang et al. [12] demonstrated that HCC with higher baseline MTT correlated with favorable clinical outcome. Relatively high radiation dose, limited coverage of the anatomy, and reproducibility are major draws backs of CTP.
Magnetic Resonance Imaging (MRI)

Over the past two decades, the use of MRI for liver imaging applications has dramatically evolved from a problem-solving tool into a more robust and comprehensive clinical tool. More recently, there have been several hardware and software advancements in MRI technology, such as higher magnetic field strength and multi-channel coil arrays, that have enabled faster MRI scanning and improved image quality. Multiple new MRI pulse sequences have been introduced, including multi-echo gradient-echo sequence for quantification of liver fat and iron overload [47], MR spectroscopy, three-point Dixon method for fat and water separation using 2D and 3D gradient-echo technique [48], high spatial resolution diffusion-weighted imaging (DWI) [49॰], and MR elastrography (MRE) for non-invasive detection and quantification of liver fibrosis. Gadoxetate disodium (Gd-EOB-DTPA) is a new hepatocyte-specific contrast agent (HSCA) that offers additional benefits over dynamic imaging for improved detection and characterization of focal lesions, as well as for allowing functional hepatic and biliary imaging [50]. The current and emerging role of DWI, MRE, and HSCAs for detection and characterization of hepatic nodules, particularly in the setting of cirrhosis, tumor therapy response evaluation, and detection and quantification of liver fibrosis, are discussed below. 

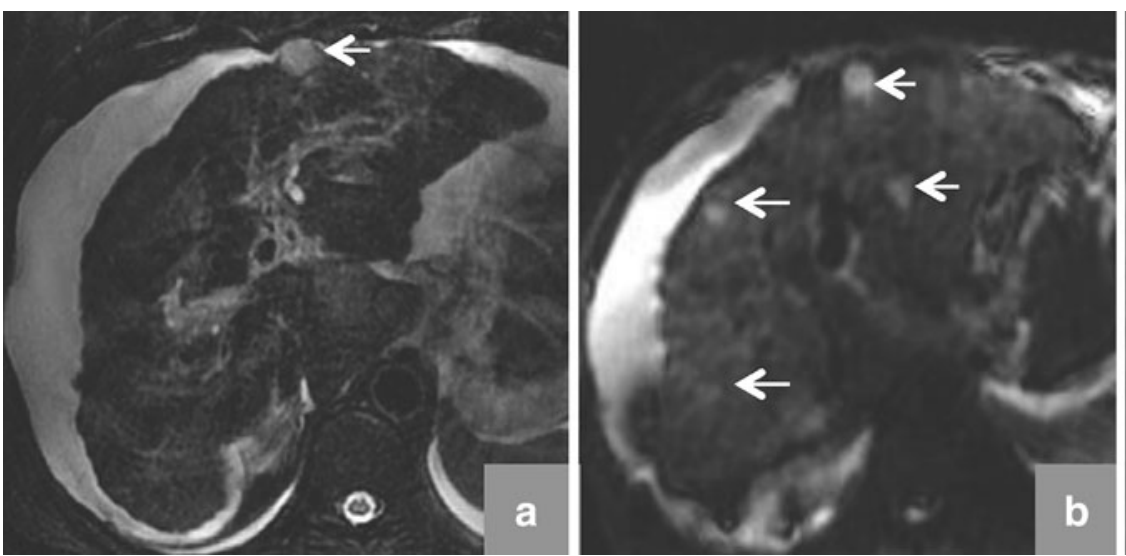

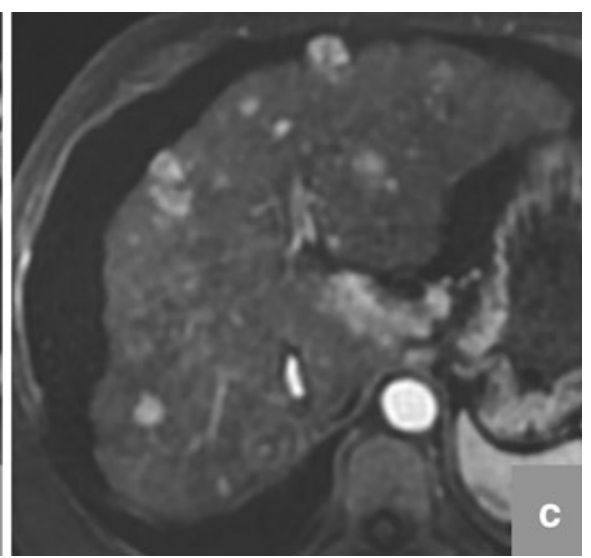

Fig. 6 Multifocal HCC in a 56-year-old-women with cirrhosis of liver: Axial T2-weighted image (a) shows a single moderately hyperintense nodule (arrow) involving the left lobe of liver. On diffusion-weighted image (b) at $b=100$, three additional (arrows) hyperintense nodules involving right and left lobes of liver were detected that were confirmed on post-gadoxetate late arterial phase T1-weighted MRI (c)

used as an imaging biomarker for the evaluation of tumor response to therapy. Tumor necrosis, loss of cell membrane integrity and increase extracellular (EC) space that occur post-treatment lead to increases in ADC value on DWI, which can often be detected before tumor size shrinkage. Therefore, DWI may be an effective early biomarker for newer targeted anticancer drugs [55].

\section{MR Elastography}

MRE is an emerging technique that offers noninvasive assessment of tissue stiffness. Conceptually, the target tissue stiffness is assessed based on its response to an external stereo mechanical force [56]. MRE utilizes an external pneumatic device that is placed onto the body wall, near the target tissue. This generates shear waves between 40 and $120 \mathrm{~Hz}$ that are propagated through the tissue deep to the transducer at a rate and velocity that is proportional to the tissue stiffness. A phase-contrast gradient-echo sequence that utilizes motion-encoding gradient is used to depict shear wave propagation. Quantitative maps for tissue stiffness can be generated by relatively rapid post-processing, and region-of-interest measurements can be taken to identify a quantitative measurement of the elastic modulus (in $\mathrm{kPa}$ ) [56].

Several investigators have investigated the utility of MRE in the assessment of liver fibrosis in patients with chronic liver disease with promising results (Fig. 7) [57, 58]. Wang et al. [59], in a meta-analysis reported that MRE has a high accuracy for the assessment of different grades of liver fibrosis as correlated with histopathology, and has the potential to replace the current gold standard of liver biopsy. Venkatesh et al. [57], reported that MRE is more accurate that serum fibrosis markers for staging liver fibrosis. Moreover, MRE is noninvasive and can be
DWI provides functional information about tumor cellularity and cell membrane integrity, and therefore can be 
Fig. 7 MR elastography: Axial post-gadolinium 2 min delayed T1-weighted image demonstrates nodular liver contour suggestive of cirrhosis; however, it is difficult to assess the severity of liver fibrosis. Axial MR elastography color map demonstrates grade-4 liver fibrosis involving both right and left lobe of liver. Note, the color scale range from 0 to $8 \mathrm{kPa}$ with highest being red color and lowest being purple color (Color figure online)

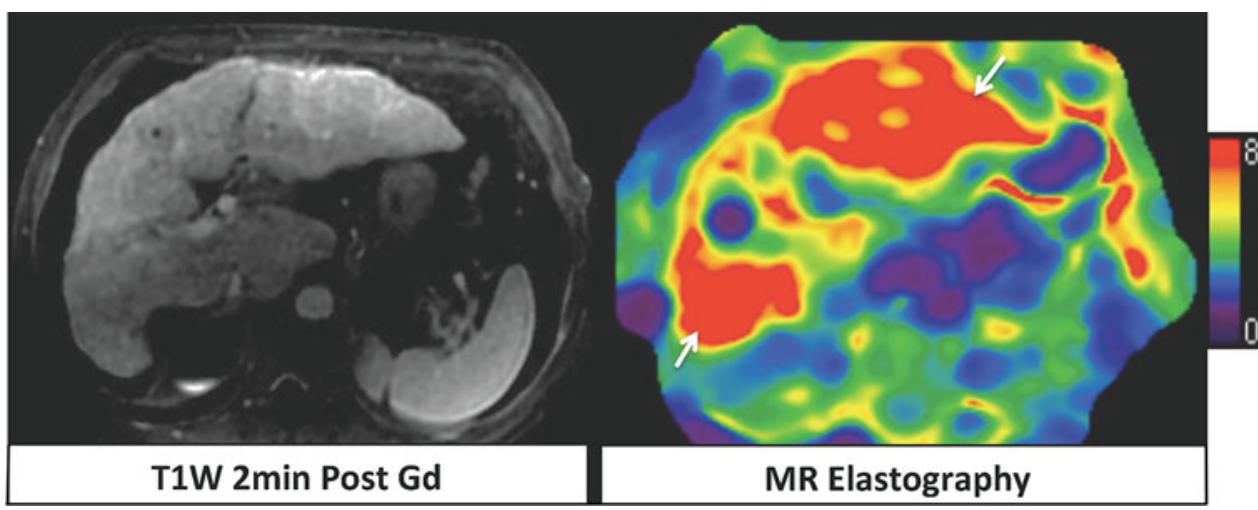

repeated to determine and monitor treatment success. With regard to focal liver lesions, preliminary studies have shown that benign hepatic tumors tend to show lower or similar stiffness to normal liver, whereas malignant lesions tends to have higher stiffness than normal liver. Venkatesh et al. [60], reported a cut-off value of $5 \mathrm{kPa}$ could be used to accurately differentiate malignant tumors from normal liver and benign lesions.

It is important to recognize that other conditions such as edema or inflammation may contribute to increased liver stiffness and confound the results [61]. In patients with non-alcoholic fatty liver disease, fatty infiltration alone does not appears to have a significant effect on hepatic stiffness. Moreover, it has been shown that disease progression to non-alcoholic steatohepatitis can be assessed on MRE even prior to the onset of clinically apparent fibrosis [62]. Limitations of MRE include the need for patient cooperation with breath-hold sequences and possible inaccurate results due to moderate or severe iron overload.

\section{Gadoxetate Disodium (Gd-EOB-DTPA)}

Traditionally used extracellular-gadolinium based contrast agents (GBCAs) provide excellent visualization of dynamic contrast-enhancement characteristics of liver lesions. However, similar to iodinated CT contrast media, they lack information specific to presence and function of hepatocytes. HSCAs on the other hand provide functional/ cellular information in addition to morphologic details. Gadoxetate (Gd-EOB-DTPA) is a HSCA with dual pharmacokinetic behavior with $50 \%$ excretion by kidneys and $50 \%$ excretion via hepatocytes into bile [63]. Initially, its behavior is similar to conventional EC-GBCAs and provides strong early enhancement enabling dynamic phase imaging. Gadoxetate is then actively taken-up by functioning hepatocytes via the OATP1B1/B3 transport proteins and excreted into biliary canaliculi via the MRP2 transport protein [64]. This active transport yields important information about liver function with superior characterization of specific lesions that possess these transport
Table 2 Gadoxetate-enhanced MRI protocol

\begin{tabular}{ll}
\hline Time $(\mathrm{s})$ & Gadoxetate-enhanced MRI protocol \\
\hline 0 & SSFSE and TruFISP localization \\
$0: 03$ & T1-W in/opposed phase \\
& Optional T2-W MRCP \\
& T1 spoiled GRE Pre Ax and Cor \\
$0: 05$ & 0.025 mmol/kg or $10 \mathrm{~mL}$ fix dose at $1 \mathrm{cc} / \mathrm{s}$ \\
& T1 spoiled GRE Ax at 25, 35, 70, $180 \mathrm{~s}$ \\
$0: 06$ & T2-W FS FSE \\
$0: 09$ & T1 spoiled GRE Ax 5 min delay \\
$0: 11$ & T1 spoiled GRE Cor 5 min delay \\
$0: 12$ & T2-W non-FS FSE \\
$0: 13$ & DWI (B50, 400, 800$)$ \\
$0: 17$ & T1 spoiled GRE Ax 20 min delay \\
$0: 26$ & T1 spoiled GRE Cor 20 min delay \\
$0: 27$ & Dynamics, 5 and 20 min subtractions \\
&
\end{tabular}

proteins. In patients with normal liver function, peak parenchymal contrast enhancement is reached within 20 min after injection and persistent enhancement is seen for more than $2 \mathrm{~h}$. This allows user to repeat data acquisition in the case of suboptimal imaging due to motion. It also allows for higher spatial resolution delayed hepatobiliary phase (HBP) imaging with higher flip angle for excellent visualization of the biliary tree [63]. From a workflow point of view, many authors have proposed reorganizing the liver MRI protocol when using gadoxetate by placing longer $\mathrm{T} 2$-weighted sequences between initial dynamic post contrast imaging and the delayed HBP imaging [63]. The protocol followed in our institute has been described in detail in Table 2.

Interpretation of gadoxetate-enhanced liver MRI can be divided into dynamic phase imaging and delayed $20 \mathrm{~min}$ HBP. Lesion behavior in the dynamic phase is similar to that with EC-GBCAs; however, because normal liver parenchyma itself takes up contrast and show progressive increase in parenchymal enhancement, the relative washout 
Fig. 8 Incidentally detected focal nodular hyperplasia (FNH) and hepatocellular adenoma (HCA) in a middleaged female on oral contraceptive drug: a axial postgadoxetate T1-weighted late arterial phase image demonstrates two homogenously hyper-enhancing lesions involving segment $4 / 8$ and segment 2 of the liver. $\mathbf{b}$ On $20 \mathrm{~min}$ hepatobiliary phase image, the segment $4 / 8$ lesion remained hyperintense to liver parenchyma (black arrow in b), suggestive of retention of gadoxetate and therefore $\mathrm{FNH}$, while segment 2 lesion showed complete washout (white arrow in b). $\mathbf{c}, \mathbf{d}$ On axial T1-weighted in and out phase images the segment 2 lesion showed loss of signal on out-phase image (white arrow in $\mathbf{d}$ ), indicating fat content and therefore confirming diagnosis of HCA
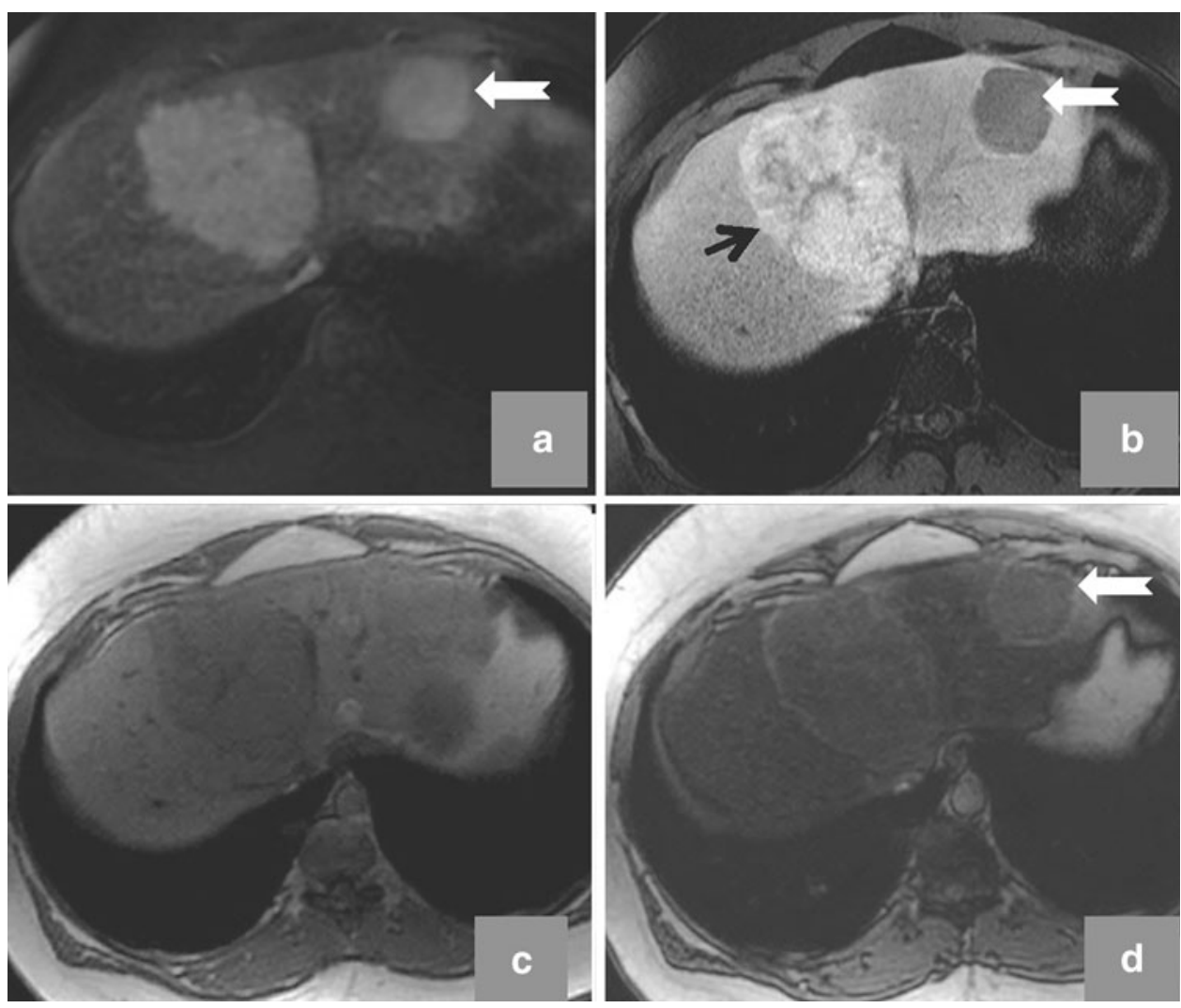

of hypervascular $\mathrm{HCC} /$ metastasis may not be obvious on portal-venous phase and is best seen on HBP imaging.

In general, on HBP imaging, lesions containing functioning hepatocytes and therefore OATP1B1/B3 transport proteins are isointense to hyperintense to the background liver, and those that lack these proteins are hypointense to the background parenchyma. Of the benign hepatocellular lesions, focal nodular hyperplasia $(\mathrm{FNH})$ appear hyperintense or isointense to the surrounding liver and can be reliably diagnosed on HBP imaging [65], whereas hepatocellular adenomas (HCA) are almost always hypointense on HBP imaging (Fig. 8). Rarely, telangiectatic/inflammatory variants of HCA may appear isointense to hyperintense on HBP, perhaps owing to variability in cellular protein expression that leads to some imaging overlap with FNH.

Liver metastases appear hypointense on HBP due to their lack of hepatocytes or functioning transport proteins. Several investigators have demonstrated improved accuracy for detection of small metastatic foci $(<1 \mathrm{~cm})$ on HBP imaging compared to CECT or CE-MRI with ECGBCAs [66, 67]. Hypointensity on HBP is not specific for metastasis, and the appearance on ancillary sequences such as T2-weighted imaging is very useful to distinguish metastasis from other hypointense benign lesions such as a cyst or hemangioma [68•].
In chronic liver disease, differentiation of hepatic nodules earlier than overt HCC (i.e. regenerative nodule [RN], low-grade dysplastic nodule [DN], high-grade DN, and early HCC) can be challenging on conventional CECT and CE-MRI, because these nodules are often small ( $<10-15 \mathrm{~mm}$ ), hiding in the multi-nodularity of cirrhosis, and may not demonstrate hyperenhancement during the hepatic arterial dominant phase. However, these premalignant/malignant lesions show changes at a cellular level (transport protein), allowing for detection on HBP imaging. In essence, benign nodules ( $\mathrm{RN}$, low grade $\mathrm{DN}$ ) retain function and show uptake of gadoxetate on HBP imaging, whereas at-risk nodules (high grade DN) and malignant nodules (HCC) have diminished or lack of hepatic function and show lack of uptake. Therefore, detection and characterization of focal lesions in cirrhotic can be reliably performed.

Recently, several authors have been investigating gadoxetate-enhanced MRI for the assessment of liver function and liver fibrosis [69]. It has been suggested that as hepatic fibrosis progresses, gadoxetate uptake mediated by OATP1B1/B3 proteins may be impaired therefore leading to a reduction in parenchymal enhancement. Therefore, gadoxetate-MRI can be useful determining hepatic functional reserve in patients undergoing hepatic resection/ transplant surgeries. The same concept can also be applied 
to evaluate post-radiation/proton therapy injury to the liver parenchyma.

\section{Conclusion}

Cross-sectional imaging plays an integral role in hepatic imaging. Knowledge and better understanding of various advances in CT and MRI technology can be used to tailor available resources for improved detection, characterization and quantification of the liver pathologies, tumor staging, and monitoring response to treatment.

\section{Compliance with Ethics Guidelines}

Conflict of Interest Mukta D. Agrawal, Sheela Agarwal, Jorge M. Fuentes-Oreego, Koichi Hayano, and Dushyant V. Sahani declare that they have no conflicts of interest.

Human and Animal Rights and Informed Consent This article does not contain any studies with human or animal subjects performed by the authors.

\section{References}

Papers of particular interest, published recently, have been highlighted as:

- Of importance,

- Of major importance

1. Singh AK, Sahani DV, Kagay CR, et al. Semiautomated MIP images created directly on 16-section multidetector CT console for evaluation of living renal donors. Radiology. 2007;244(2): 583-90.

2. Singh AK, Sahani DV, Blake MA, et al. Assessment of pancreatic tumor resectability with multidetector computed tomography: semiautomated console-generated images versus dedicated workstation-generated images. Acad Radiol. 2008;15(8):1058-68.

3. Singh AK, Hiroyuki Y, Sahani DV. Advanced postprocessing and the emerging role of computer-aided detection. Radiol Clin $\mathrm{N}$ Am. 2009;47(1):59-77.

4. Motosugi U, Ichikawa $\mathrm{T}$, Sou $\mathrm{H}$, et al. Multi-organ perfusion CT in the abdomen using a 320-detector row CT scanner: preliminary results of perfusion changes in the liver, spleen, and pancreas of cirrhotic patients. Eur J Radiol. 2012;81(10):2533-7.

5. Kanda T, Yoshikawa T, Ohno Y, et al. Perfusion measurement of the whole upper abdomen of patients with and without liver diseases: initial experience with 320-detector row CT. Eur J Radiol. 2012;81(10):2470-5.

6. Ichikawa T, Motosugi U, Morisaka H, et al. Volumetric low-tubevoltage CT imaging for evaluating hypervascular hepatocellular carcinoma; effects on radiation exposure, image quality, and diagnostic performance. Jpn J Radiol. 2013;31(8):521-9.

7. Okada M, Kim T, Murakami T. Hepatocellular nodules in liver cirrhosis: state of the art CT evaluation (perfusion $\mathrm{CT} /$ volume helical shuttle scan/dual-energy CT, etc.). Abdom Imaging. 2011;36(3):273-81.
8. Yu MH, Lee JM, Yoon J-H, et al. Low tube voltage intermediate tube current liver MDCT: sinogram-affirmed iterative reconstruction algorithm for detection of hypervascular hepatocellular carcinoma. Am J Roentgenol. 2013;201(1):23-32.

9. Singh S, Kalra MK, Shenoy-Bhangle AS, et al. Radiation dose reduction with hybrid iterative reconstruction for pediatric $\mathrm{CT}$. Radiology. 2012;263(2):537-46.

10. - Singh S, Kalra MK, Do S, et al. Comparison of hybrid and pure iterative reconstruction techniques with conventional filtered back projection: dose reduction potential in the abdomen. J Comput Assist Tomogr. 2012;36(3):347-53. This articles has demonstrated that model-based reconstruction technique permits diagnostic quality ultra low-dose CT Abdomen.

11. Silva AC, Lawder HJ, Hara A, et al. Innovations in CT dose reduction strategy: application of the adaptive statistical iterative reconstruction algorithm. Am J Roentgenol. 2010;194(1):191-9.

12. Jiang $\mathrm{T}$, Kambadakone A, Kulkarni NM, et al. Monitoring response to antiangiogenic treatment and predicting outcomes in advanced hepatocellular carcinoma using image biomarkers, CT perfusion, tumor density, and tumor size (RECIST). Investig Radiol. 2012;47(1):11-7.

13. Yeh BM, Shepherd JA, Wang ZJ, et al. Dual-energy and low-kVp CT in the abdomen. Am J Roentgenol. 2009;193(1):47-54.

14. De Cecco CN, Darnell A, Rengo M, et al. Dual-energy CT: oncologic applications. Am J Roentgenol. 2012;199(5 Suppl): S98-105.

15. Schindera ST, Nelson RC, Mukundan S Jr, et al. Hypervascular liver tumors: low tube voltage, high tube current multi-detector row CT for enhanced detection-phantom study. Radiology. 2008;246(1):125-32.

16. Marin D, Nelson RC, Samei E, et al. Hypervascular liver tumors: low tube voltage, high tube current multidetector $\mathrm{CT}$ during late hepatic arterial phase for detection-initial clinical experience. Radiology. 2009;251(3):771-9.

17. Nakayama Y, Awai K, Funama Y, et al. Abdominal CT with low tube voltage: preliminary observations about radiation dose, contrast enhancement, image quality, and noise. Radiology. 2005;237(3):945-51.

18. Kalra MK, Maher MM, Toth TL, et al. Techniques and applications of automatic tube current modulation for CT. Radiology. 2004;233(3):649-57.

19. Kalra MK, Rizzo SMR, Novelline RA. Reducing radiation dose in emergency computed tomography with automatic exposure control techniques. Emerg Radiol. 2005;11(5):267-74.

20. Raman SP, Johnson PT, Deshmukh S, et al. CT dose reduction applications: available tools on the latest generation of CT scanners. J Am Coll Radiol. 2013;10(1):37-41.

21. Marin D, Nelson RC, Schindera ST, et al. Low-tube-voltage, high-tube-current multidetector abdominal CT: improved image quality and decreased radiation dose with adaptive statistical iterative reconstruction algorithm-initial clinical experience. Radiology. 2010;254(1):145-53.

22. - Kulkarni NM, Pinho DF, Kambadakone AR, et al. Emerging technologies in CT-radiation dose reduction and dual-energy CT. Semin Roentgenol. 2013;48(3):192-202. This review article details various $C T$ parameters that can be adjusted to reduce radiation exposure.

23. Kalra MK, Woisetschläger M, Dahlström N, et al. Radiation dose reduction with Sinogram Affirmed Iterative Reconstruction technique for abdominal computed tomography. J Comput Assist Tomogr. 2012;36(3):339-46.

24. Deák Z, Grimm JM, Treitl M, et al. Filtered back projection, adaptive statistical iterative reconstruction, and a model-based iterative reconstruction in abdominal CT: an experimental clinical study. Radiology. 2013;266(1):197-206. 
25. Graser A, Johnson TRC, Chandarana H, et al. Dual energy CT: preliminary observations and potential clinical applications in the abdomen. Eur Radiol. 2009;19(1):13-23.

26. • Heye T, Nelson RC, Ho LM, et al. Dual-energy CT applications in the abdomen. Am J Roentgenol. 2012;199(5 Suppl):S64-70. This article provide detail about applications of DECT in the abdomen.

27. Lee SH, Lee JM, Kim KW, et al. Dual-energy computed tomography to assess tumor response to hepatic radiofrequency ablation: potential diagnostic value of virtual noncontrast images and iodine maps. Investig Radiol. 2011;46(2):77-84.

28. Graser A, Johnson TRC, Hecht EM, et al. Dual-energy CT in patients suspected of having renal masses: can virtual nonenhanced images replace true nonenhanced images? Radiology. 2009;252(2):433-40.

29. Ho LM, Marin D, Neville AM, et al. Characterization of adrenal nodules with dual-energy CT: can virtual unenhanced attenuation values replace true unenhanced attenuation values? Am J Roentgenol. 2012;198(4):840-5.

30. Mahgerefteh S, Blachar A, Fraifeld S, et al. Dual-energy derived virtual nonenhanced computed tomography imaging: current status and applications. Semin Ultrasound CT MRI. 2010;31(4):321-7.

31. Yu L, Leng S, McCollough CH. Dual-energy CT-based monochromatic imaging. Am J Roentgenol. 2012;199(5 Suppl):S9-15.

32. Altenbernd J, Heusner TA, Ringelstein A, et al. Dual-energy-CT of hypervascular liver lesions in patients with HCC: investigation of image quality and sensitivity. Eur Radiol. 2011;21(4):738-43.

33. Robinson E, Babb J, Chandarana $\mathrm{H}$, et al. Dual source dual energy MDCT: comparison of $80 \mathrm{kVp}$ and weighted average 120 $\mathrm{kVp}$ data for conspicuity of hypo-vascular liver metastases. Investig Radiol. 2010;45(7):413-8.

34. Mendonca PRS, Lamb P, Sahani DV. A flexible method for multi-material decomposition of dual-energy CT images. IEEE Trans Med Imaging. 2013. doi:10.1109/TMI.2013.2281719.

35. Tu R, Xia L-P, Yu A-L, et al. Assessment of hepatic functional reserve by cirrhosis grading and liver volume measurement using CT. World J Gastroenterol. 2007;13(29):3956-61.

36. Pech M, Mohnike K, Wieners G, et al. Radiotherapy of liver metastases. Comparison of target volumes and dose-volume histograms employing CT- or MRI-based treatment planning. Strahlenther Onkol Organ Dtsch Röntgengesellschaft Al. 2008;184(5):256-61.

37. Kele PG, de Jong KP, van der Jagt EJ. Increase in volume of ablation zones during follow-up is highly suggestive of ablation site recurrence in colorectal liver metastases treated with radiofrequency ablation. J Vasc Interv Radiol. 2012;23(4):537-44.

38. Heussel CP, Meier S, Wittelsberger S, et al. Follow-up CT measurement of liver malignoma according to RECIST and WHO vs. volumetry. RöFo Fortschritte Auf Dem Geb Röntgenstrahlen Nukl. 2007;179(9):958-64.

39. Miles KA, Hayball M, Dixon AK. Colour perfusion imaging: a new application of computed tomography. Lancet. 1991;337(8742): 643-5.

40. Ronot M, Asselah T, Paradis V, et al. Liver fibrosis in chronic hepatitis $\mathrm{C}$ virus infection: differentiating minimal from intermediate fibrosis with perfusion CT. Radiology. 2010;256(1):135-42.

41. Hashimoto K, Murakami T, Dono K, et al. Assessment of the severity of liver disease and fibrotic change: the usefulness of hepatic CT perfusion imaging. Oncol Rep. 2006;16(4):677-83.

42. Van Beers BE, Leconte I, Materne R, et al. Hepatic perfusion parameters in chronic liver disease: dynamic CT measurements correlated with disease severity. Am J Roentgenol. 2001;176(3): 667-73.

43. Sahani DV, Holalkere N-S, Mueller PR, et al. Advanced hepatocellular carcinoma: CT perfusion of liver and tumor tissueinitial experience. Radiology. 2007;243(3):736-43.
44. Zhu AX, Holalkere NS, Muzikansky A, et al. Early antiangiogenic activity of bevacizumab evaluated by computed tomography perfusion scan in patients with advanced hepatocellular carcinoma. Oncologist. 2008;13(2):120-5.

45. • Jiang T, Zhu AX, Sahani DV. Established and novel imaging biomarkers for assessing response to therapy in hepatocellular carcinoma. J Hepatol. 2013;58(1):169-77. This review article describes various currently used and emerging imaging biomarkers for assessing response to hepatic malignancy.

46. Miles KA. Perfusion CT for the assessment of tumour vascularity: which protocol? Br J Radiol. 2003;76 Spec No 1:S36-42.

47. Henninger B, Kremser C, Rauch S, et al. Evaluation of MR imaging with $\mathrm{T} 1$ and $\mathrm{T} 2 *$ mapping for the determination of hepatic iron overload. Eur Radiol. 2012;22(11):2478-86.

48. Reeder SB, Sirlin CB. Quantification of liver fat with magnetic resonance imaging. Magn Reson Imaging Clin N Am. 2010;18(3): 337-57, ix.

49. - Galea N, Cantisani V, Taouli B. Liver lesion detection and characterization: role of diffusion-weighted imaging. J Magn Reson Imaging. 2013;37(6):1260-76. This review article gives detailed description of DWI and its applications for hepatic imaging.

50. Cruite I, Schroeder M, Merkle EM, et al. Gadoxetate disodiumenhanced MRI of the liver: part 2, protocol optimization and lesion appearance in the cirrhotic liver. Am J Roentgenol. 2010;195(1):29-41.

51. Parikh T, Drew SJ, Lee VS, et al. Focal liver lesion detection and characterization with diffusion-weighted MR imaging: comparison with standard breath-hold T2-weighted imaging. Radiology. 2008;246(3):812-22.

52. Hardie AD, Naik M, Hecht EM, et al. Diagnosis of liver metastases: value of diffusion-weighted MRI compared with gadolinium-enhanced MRI. Eur Radiol. 2010;20(6):1431-41.

53. Kandpal H, Sharma R, Madhusudhan KS, et al. Respiratorytriggered versus breath-hold diffusion-weighted MRI of liver lesions: comparison of image quality and apparent diffusion coefficient values. Am J Roentgenol. 2009;192(4):915-22.

54. Nasu K, Kuroki Y, Sekiguchi R, et al. The effect of simultaneous use of respiratory triggering in diffusion-weighted imaging of the liver. Magn Reson Med Sci Off J Jpn Soc Magn Reson Med. 2006;5(3):129-36.

55. Sahani DV, Jiang $\mathrm{T}$, Hayano $\mathrm{K}$, et al. Magnetic resonance imaging biomarkers in hepatocellular carcinoma: association with response and circulating biomarkers after sunitinib therapy. J Hematol Oncol. 2013;6:51. doi:10.1186/1756-8722-6-51.

56. Venkatesh SK, Yin M, Ehman RL. Magnetic resonance elastography of liver: technique, analysis, and clinical applications. J Magn Reson Imaging. 2013;37(3):544-55.

57. Venkatesh SK, Wang G, Lim SG, et al. Magnetic resonance elastography for the detection and staging of liver fibrosis in chronic hepatitis B. Eur Radiol. 2013. doi:10.1007/s00330-0132978-8.

58. Lee YJ, Lee JM, Lee JE, et al. MR elastography for noninvasive assessment of hepatic fibrosis: reproducibility of the examination and reproducibility and repeatability of the liver stiffness value measurement. J Magn Reson Imaging. 2013. doi:10.1002/jmri. 24147.

59. Wang Q-B, Zhu H, Liu H-L, et al. Performance of magnetic resonance elastography and diffusion-weighted imaging for the staging of hepatic fibrosis: a meta-analysis. Hepatology (Baltim Md). 2012;56(1):239-47.

60. Venkatesh SK, Yin M, Glockner JF, et al. MR elastography of liver tumors: preliminary results. Am J Roentgenol. 2008;190(6): 1534-40.

61. Faria SC, Ganesan K, Mwangi I, et al. MR imaging of liver fibrosis: current state of the art. Radiogr Rev Publ Radiol Soc N Am Inc. 2009;29(6):1615-35. 
62. Chen J, Talwalkar JA, Yin M, et al. Early detection of nonalcoholic steatohepatitis in patients with nonalcoholic fatty liver disease by using MR elastography. Radiology. 2011;259(3):749-56.

63. Ringe KI, Husarik DB, Sirlin CB, et al. Gadoxetate disodiumenhanced MRI of the liver: part 1, protocol optimization and lesion appearance in the noncirrhotic liver. Am J Roentgenol. 2010;195(1):13-28.

64. Nassif A, Jia J, Keiser M, et al. Visualization of hepatic uptake transporter function in healthy subjects by using gadoxetic acidenhanced MR imaging. Radiology. 2012;264(3):741-50.

65. Purysko AS, Remer EM, Coppa CP, et al. Characteristics and distinguishing features of hepatocellular adenoma and focal nodular hyperplasia on gadoxetate disodium-enhanced MRI. Am J Roentgenol. 2012;198(1):115-23.

66. Hammerstingl R, Huppertz A, Breuer J, et al. Diagnostic efficacy of gadoxetic acid (Primovist)-enhanced MRI and spiral CT for a therapeutic strategy: comparison with intraoperative and histopathologic findings in focal liver lesions. Eur Radiol. 2008;18(3):457-67.

67. Zech CJ, Herrmann KA, Reiser MF, et al. MR imaging in patients with suspected liver metastases: value of liver-specific contrast agent Gd-EOB-DTPA. Magn Reson Med Sci Off J Jpn Soc Magn Reson Med. 2007;6(1):43-52.

68. - Frydrychowicz A, Lubner MG, Brown JJ, et al. Hepatobiliary MR imaging with gadolinium-based contrast agents. J Magn Reson Imaging. 2012;35(3):492-511. This review article explains details about protocol optimization and interpretation of gadoxetate-enhanced MRI.

69. Nilsson H, Blomqvist L, Douglas L, et al. Gd-EOB-DTPAenhanced MRI for the assessment of liver function and volume in liver cirrhosis. Br J Radiol. 2013;86(1026):20120653. doi:10. 1259/bjr.20120653. 